\title{
Excessive Contrast Medium Leakage in Hyperperfusion Syndrome
}

\author{
P. Mordasini · C. Brekenfeld · C. Fung • D. D. Do · \\ G. Schroth · J. Gralla
}

Received: 14 October 2010 / Accepted: 23 December 2010 / Published online: 4 February 2011

(C) Urban \& Vogel 2011

\section{Introduction}

Cerebral hyperperfusion syndrome is a rare but serious complication after revascularization of stenosis of blood vessels supplying the brain and has been frequently described following carotid artery stenting. Impaired cerebral autoregulation and post-revascularization changes in cerebral hemodynamics are the most important mechanisms involved in the development of the syndrome. Clinical symptoms often include the triad of headache, seizures and transient focal neurological deficits in the absence of cerebral ischemia. The incidence of hyperperfusion injury is estimated to be $1.1-6.8 \%$, with mortality rates ranging from $3-26 \%$ when intracranial hemorrhage (ICH) is present. However, imaging in these patients is often uneventful and edema is frequently found in the affected areas of the brain.

The literature on hyperperfusion syndrome of the posterior circulation and imaging findings is rare. A case of hyperperfusion syndrome following recanalization of an occluded left subclavian artery (LSA) with an extraordinary extravasation of contrast medium and delayed occurrence of ICH is presented.

P. Mordasini, MD · C. Brekenfeld, MD · G. Schroth, MD ·

J. Gralla, MD MSc $(\bowtie)$

Department of Interventional and Diagnostic Neuroradiology,

University of Bern, Inselspital, Freiburgstrasse 4,

3010 Bern, Switzerland

e-mail: jan.gralla@insel.ch

C. Fung, MD

Department of Neurosurgery, Bern, Switzerland

D. D. Do, MD

Department of Angiology, Inselspital,

University of Bern, Bern, Switzerland

\section{Case Report}

A 56-year-old female patient presented with symptoms of intermittent claudication of the left arm and subclavian steal syndrome under physical stress. The pre-interventional clinical examination using computed tomography angiography (CTA), digital subtraction angiography (DSA) and ultrasound confirmed the diagnosis of LSA occlusion proximal to the origin of the left vertebral artery (LVA) without any further high-grade stenosis of the other cervical arteries supplying the brain (Fig. 1). The retrograde flow in the LVA confirmed a subclavian steal phenomenon. The pre-interventional CTA and DSA ruled out any further intracranial vascular pathologies (e. g. aneurysms and arterio-venous malformation).

The arterial blood pressure measured at the right arm was $130 / 95 \mathrm{mmHg}$ prior to the intervention. Pre-interventional antiaggregation was established by a daily dosage of $100 \mathrm{mg}$ aspirin and $75 \mathrm{mg}$ clopidogrel and peri-interventional anticoagulation was induced by the additional application of $5000 \mathrm{U}$ of heparin.

Under local anesthesia an 8 French (F) sheath was introduced into the right femoral artery and a $6 \mathrm{~F}$ sheath into the left brachial artery. Digital subtraction angiography of the right subclavian artery (RSA) illustrates the retrograde flow in the LVA (Fig. 2a). The angiogram obtained via a $6 \mathrm{~F}$ diagnostic catheter (Terumo, Japan) introduced into the left brachial artery illustrates the complete occlusion of the LSA (Fig. 2b). The occlusion site was passed from the brachial approach using a straight tip 0.35 inch guidewire (Terumo, Japan). The wire was caught in the thoracic aorta by a snare introduced from the inguinal approach and a long $8 \mathrm{~F}$ sheath (destination sheath, Terumo, Japan) was introduced crossing the site of occlusion. A balloon-mounted stent (Scuba $6 \times 28 \mathrm{~mm}$, Invatec, Italy) was placed achieving a good recanalization result (Fig. 2c). A total 

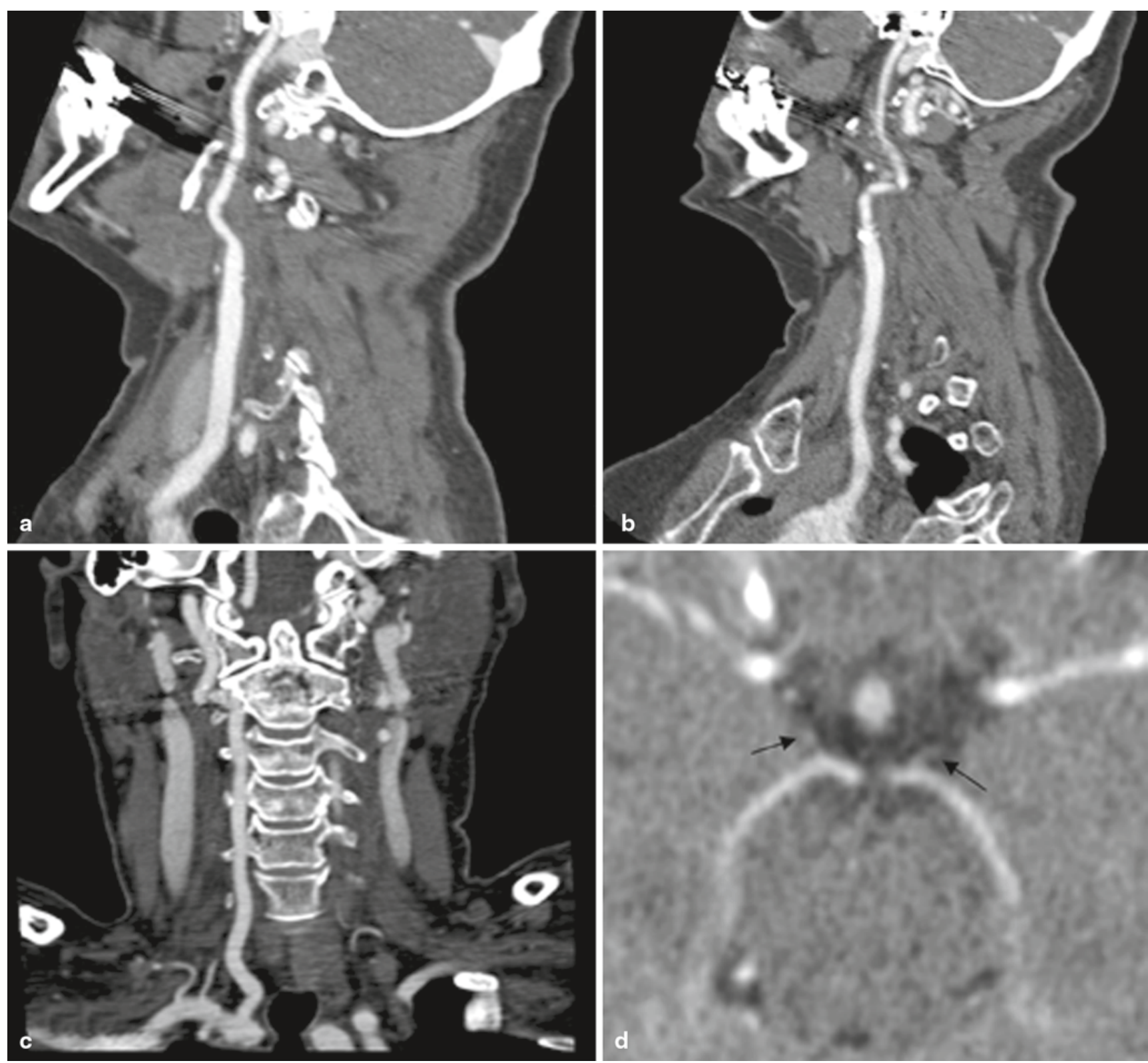

Fig. 1 Pre-interventional computed tomography angiography (CTA) with curved reconstruction showing the right carotid artery (a), the left carotid artery (b), and the right vertebral artery (c), without any

concomitant high-grade stenosis. The intracranial pre-interventional CTA illustrates small posterior communication arteries (arrows) on both sides (d)

of $170 \mathrm{ml}$ low osmolar iodine contrast medium (Iopamiro 300, Bracco, USA) was used.

Immediately after recanalization, the patient complained of severe headache and became agitated. The intraarterial blood pressure revealed a raised systolic blood pressure of $205 \mathrm{mmHg}$. The blood pressure was medicinally reduced and kept within narrow margins below $140 \mathrm{mmHg}$ systolic blood pressure. The patient showed no neurological deficits, in particular no signs of cerebellar dysfunction. A CT scan was performed 25 min after recanalization (Fig. 3) and showed an extraordinary hyperdense signal alteration bilaterally and symmetrically in the cerebellar hemispheres, following the cortex in the temporo-occipital lobe predominantly on the left side and presumably within the adjacent subarachnoidal space. The parenchymal hyperdensity without any mass-occupying effect in conjunction with the severe headache immediately after recanalization was interpreted as a massive contrast agent extravasation.

The MRI $6.5 \mathrm{~h}$ after the intervention including diffusion-weighted imaging (DWI), T2-weighted imaging, susceptibility-weighted imaging (SWI) and T1-weighted pre-contrast and post-contrast imaging demonstrated edema in the corresponding cerebellar and cerebral areas (Fig. 4). No intracranial hemorrhage and ischemia were found. The 

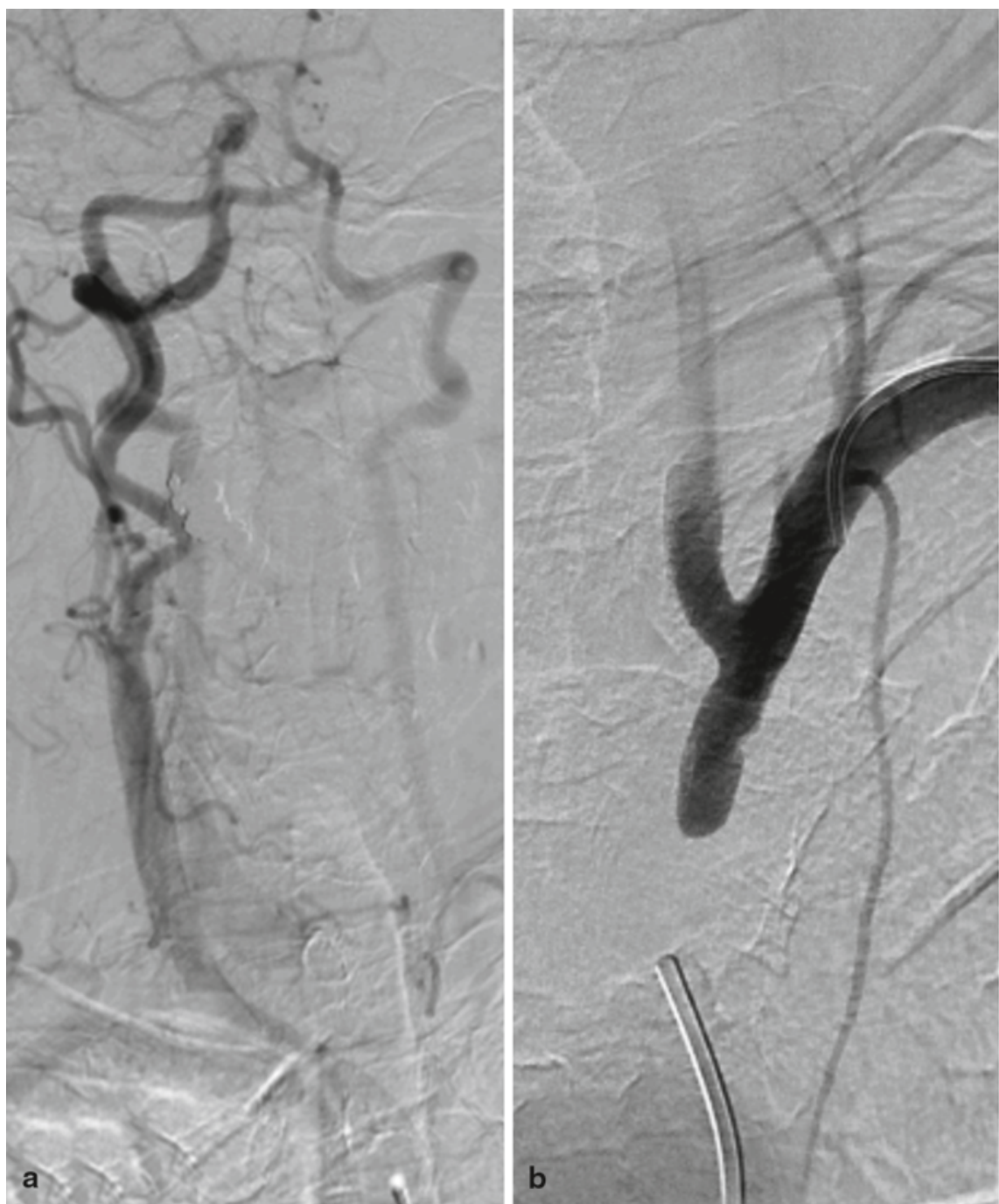

Fig. 2 Digital subtraction angiography (DSA) of the right subcalvian artery illustrates a subclavian steal syndrome with retrograde flow in the left vertebral artery (a), the retrograde angiography of the left subclavian artery shows a long occlusion of the artery, the distal guider

gadolinium-enhanced T1-weighted images did not illustrate any pathological enhancement. The patient was referred to the intensive care unit (ICU) for arterial blood pressure monitoring. The follow-up CT performed $23 \mathrm{~h}$ later illustrated a complete disappearance of the parenchymal hyperdensities with some residual edema in the left occipital lobe (Fig. 5a and b).

Clopidogrel and aspirin medication was stopped after the intervention. After an uneventful clinical course in the ICU with normal blood pressure and no further antihypertensive medication for 2 days, the patient was returned to the ward. However, $63 \mathrm{~h}$ after the intervention the patient showed marked neurological deterioration and became aphasic. The control CT illustrated a mass-occupying intracerebral hemorrhage in the left occipital and temporal lobes with midline shift and intraventricular hemorrhage

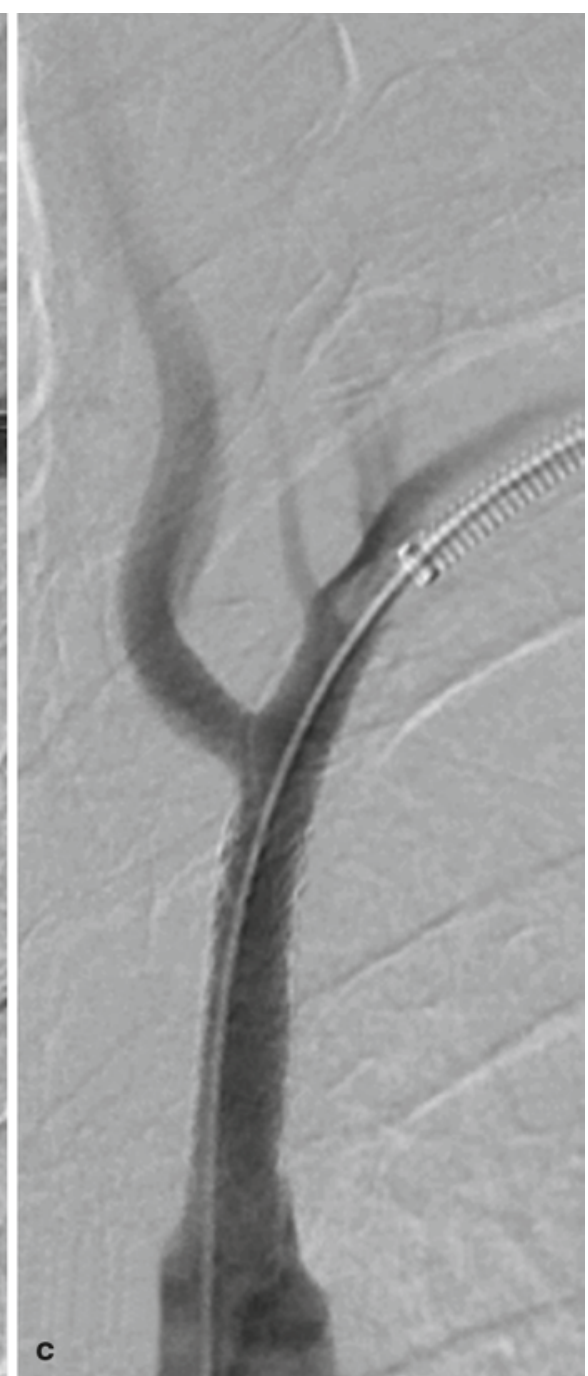

is placed in the origin at the aortic arch (b), postinterventional angiography illustrates a good recanalization with antegrade flow in the left vertebral artery $(\mathbf{c})$

(Fig. $5 \mathrm{c}$ and d). The patient recovered well after surgery and was sent to rehabilitation with a minor speech deficit and quadrantanopsia.

\section{Discussion}

Cerebral hyperperfusion syndrome is a serious complication of craniocervical revascularization and is well-described after carotid artery endarterectomy and stenting. Two synergistic mechanisms may be responsible for the development of the syndrome: impaired cerebral autoregulation and postoperatively elevated systemic blood pressure. It is theorized that the capillary bed beyond the stenosis is prone to breakdown of the blood-brain barrier after an increase of blood flow due to impaired autoregulation. However, reports 

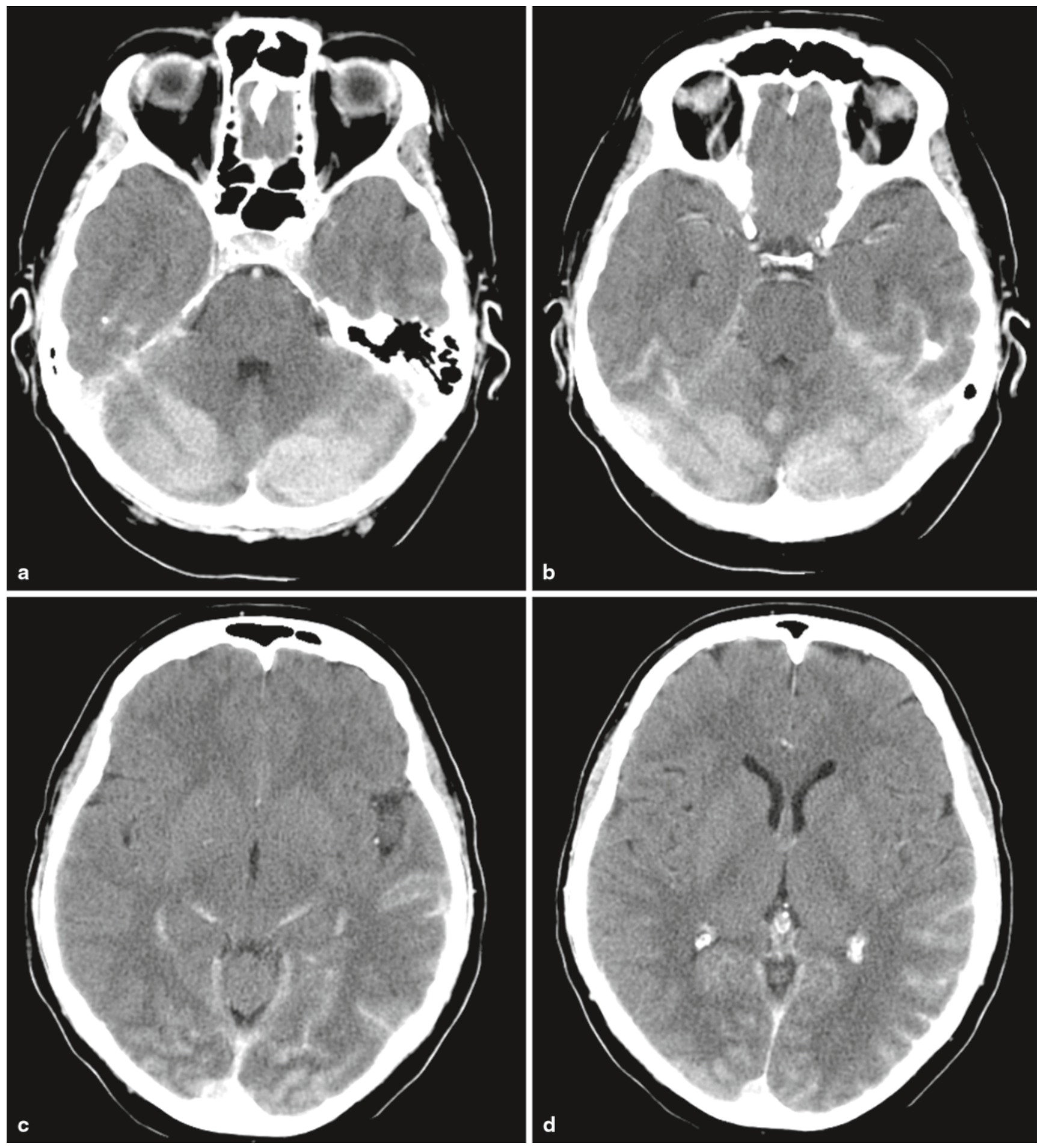

Fig. 3 Postinterventional CT performed $25 \mathrm{~min}$ after recanalization of the LSA shows extensive areas of hyperdense signal symmetrically distributed in both cerebellar hemispheres including white matter and

of this phenomenon after recanalization of the subclavian artery [1] or vertebral artery [2-4] are limited.

The clinical symptoms in conjunction with increased systemic blood pressure confirm the diagnosis of hyperperfusion syndrome in the presented case. The extraordinary parenchy-

cortex $(\mathbf{a}, \mathbf{b})$. A linear hyperdense signal is seen following the occipital and temporal cortex and subarachnoidal space $(\mathbf{c}, \mathbf{d})$

mal hyperdensity in CT can mimic laminar intraparenchymal hemorrhaging although no relevant mass-occupying effect was illustrated. The finding was consistent with a massive extravasation of iodine contrast agent immediately after stenting and is most likely the consequence of breakdown of 

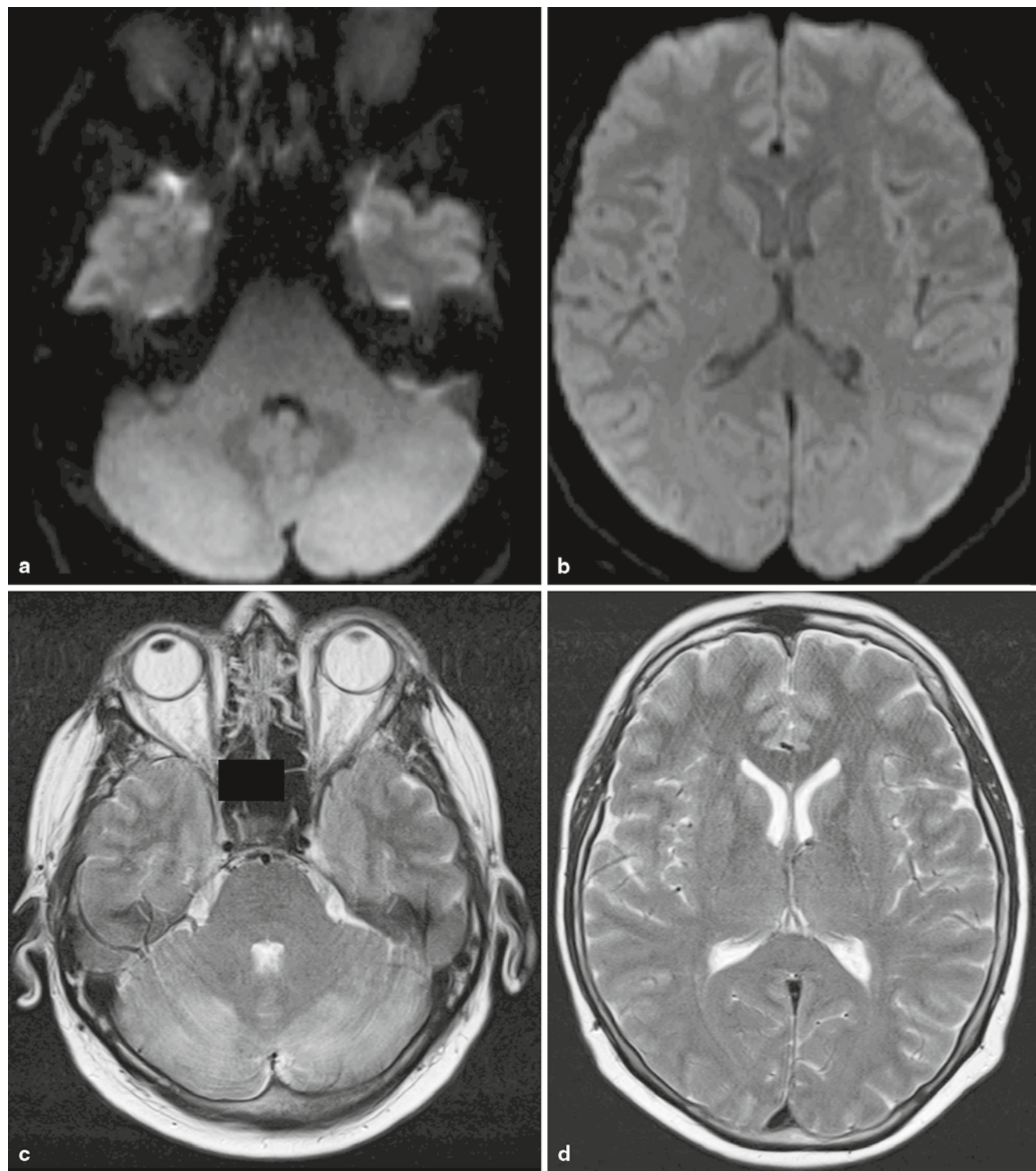

Fig. 4 The postinterventional MRI $6.5 \mathrm{~h}$ after intervention in digitalweighted imaging (DWI) shows no signs of infarction or hemorrhage $(\mathbf{a}, \mathbf{b})$. The T2-weighted images show hyperintense signals in both

cerebellar hemispheres and subtle changes in the left occipital lobe $(\mathbf{c}, \mathbf{d})$

the blood-brain barrier. The MRI ruled out any intracranial hemorrhage but illustrated brain areas affected by edema. Interestingly, at this point ( $6.5 \mathrm{~h}$ after intervention) no further extravasation of gadolinium-based contrast was found. The

reduced extravasation of contrast agent in the post-interventional course might be explained either by differences for contrast agents to pass the damaged blood-brain barrier or by decrease of systemic blood pressure. 

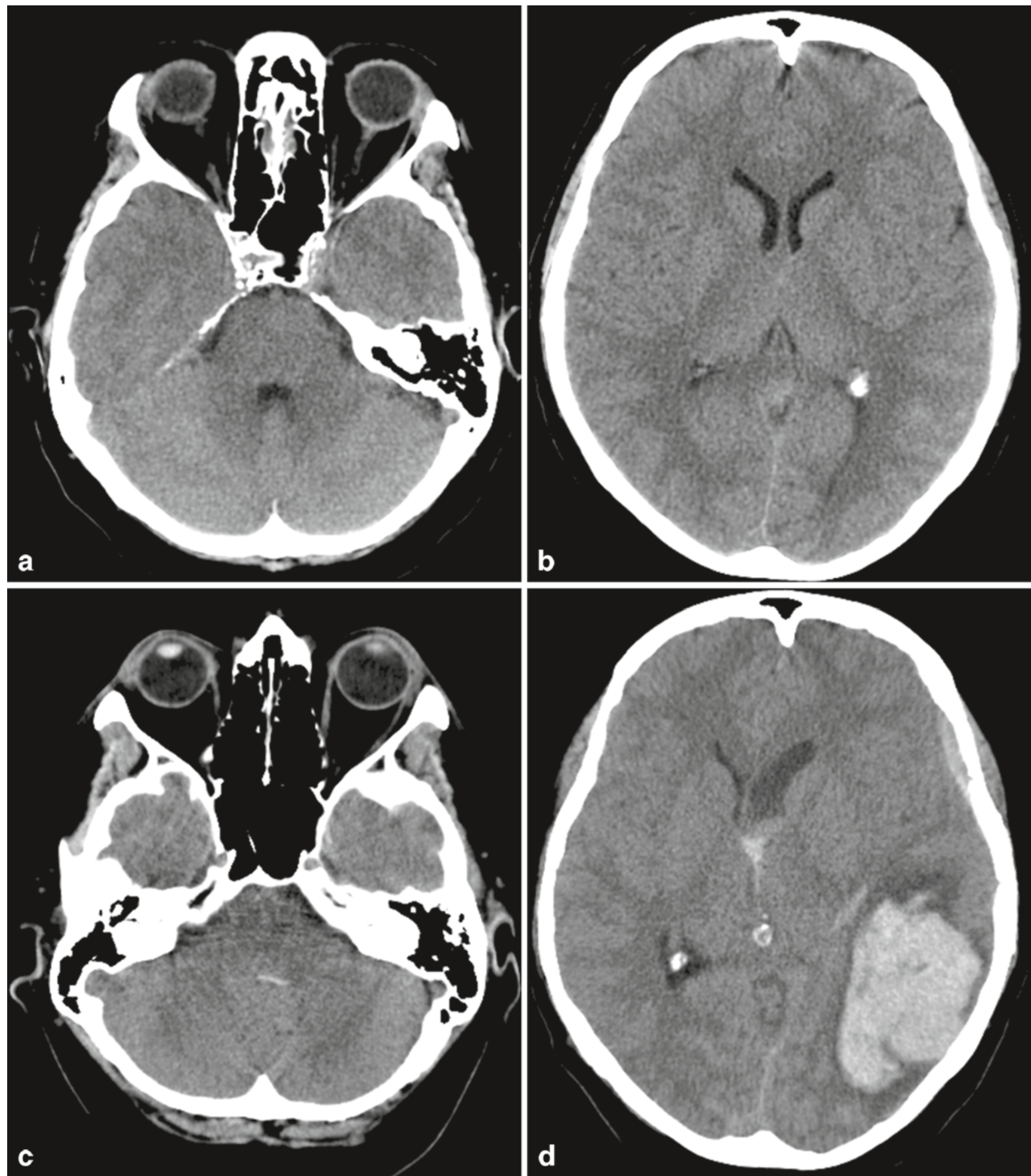

Fig. 5 The unenhanced CT performed $23 \mathrm{~h}$ after intervention shows some edema in the occipital lobes, the hyperdense signal in the infratentorial and supratentorial spaces however has completely diminished (a, b), the CT after acute neurological deterioration performed $63 \mathrm{~h}$

Moreover it is unlikely that the parenchymal hemorrhage was the result of a reperfusion lesion into an area of tissue infarction as both CT and MRI imaging illustrated

after intervention illustrates some hemorrhaging in the fourth ventricle but the cerebellar parenchyma is unaffected (c), mass-occupying intracerebral hemorrhage in the left occipital and temporal lobe with intraventricular hemorrhage and midline shift (d)

no ischemic lesions. Remarkably, parenchymal hemorrhage occurred after a delay of $63 \mathrm{~h}$ after intervention into the left temporo-occipital lobe and despite normal systolic 
blood pressure during this period. The hemorrhage therefore occurred in a brain area previously affected by extravasation of contrast agent and edema. This finding is in concordance with previous case reports [5-7]. It is hypothesized that contrast agent extravasation in the course of cerebral hyperperfusion syndrome indicates more serious damage of the blood-brain barrier and that these areas are at increased risk for intraparenchymal hemorrhage.

\section{Conclusion}

Cerebral and cerebellar hyperperfusion syndrome complicated by hemorrhage may occur after recanalization of the subclavian artery. Excessive extravasation of iodine contrast agent can mimic parenchymal hemorrhage and is likely to be a consequence of breakdown of the blood-brain barrier. Despite stringent blood pressure control intracranial hemorrhage might occur.

Conflict of Interest Statement The authors declare that they have no conflict of interest.

\section{References}

1. Salerno JL, Vitek J. Fatal cerebral hemorrhage early after subclavian artery endovascular therapy. AJNR Am J Neuroradiol. 2005;26:183-5.

2. Zhang R, Zhou G, Xu G, Liu X. Posterior circulation hyperperfusion syndrome after bilateral vertebral artery intracranial stenting. Ann Vasc Surg. 2009;23:686-5.

3. Rezende MT, Spelle L, Mounayer C, Piotin M, Abud DG, Moret J. Hyperperfusion syndrome after stenting for intracranial vertebral stenosis. Stroke. 2006;37:e12-e14.

4. Bando K, Satoh K, Matsubara S, Nakatani M, Nagahiro S. Hyperperfusion phenomenon after percutaneous transluminal angioplasty for atherosclerotic stenosis of the intracranial vertebral artery. Case report. J Neurosurg. 2001;94:826-30.

5. Takayama K, Nakagawa H, Iwasaki S, Taoka T, Wada T, Myouchin K, Sakamoto M, Fukusumi A, Kichikawa K. Cerebral hemorrhage with angiographic extravasation immediately after carotid artery stenting. Radiat Med. 2007;25(7):359-63.

6. Toh CH, Wong HF, Lin TK, Ng SH. Abnormal enhancement on imaging studies preceding hyperperfusion syndrome. Case illustration. J Neurosurg. 2006;105:932.

7. Sharma P, Poppe AY, Eesa M, Steffanhagan N, Hudon M, Morrish $\mathrm{W}$. Extravasating contrast material on angiography following carotid angioplasty and stenting: not necessarily subarachnoid hemorrhage. J Neuroimaging. 2010;20:180-82. 